\title{
Brain iron homeostasis, the choroid plexus, and localization of iron transport proteins
}

\author{
Tracey A. Rouault • De-Liang Zhang • \\ Suh Young Jeong
}

Received: 31 July 2009 / Accepted: 4 August 2009 /

Published online: 23 October 2009

(C) The Author(s) 2009. This article is published with open access at Springerlink.com

\begin{abstract}
Maintenance of appropriate iron homeostasis in the brain is important, but the mechanisms involved in brain iron uptake are incompletely understood. Here, we have analyzed where messenger RNAs that encode iron transport proteins are expressed in the brain, using the Allen Brain atlas, and we conclude that several important iron transporters are highly expressed in the choroid plexus. Based on recent estimates of the surface area of the choroid plexus and on MRI imaging studies of manganese uptake in the brain, we propose that the choroid plexus may have a much greater role than has been previously appreciated in brain iron transport.
\end{abstract}

Keywords Choroid plexus - Iron transport - Blood brain barrier - Blood-CSF barrier Ferroportin · Ceruloplasmin

It has long been known that iron deficiency has a significant negative impact on brain development and cognition, and recent studies indicate that both the striatal dopaminergic-opiate system and the cholinergic systems are adversely affected by iron deficiency (Youdim 2008). Nutritional iron deficiency has a significant impact on infant and child health in many developing countries, where it is one of the major public health problems (Walker et al. 2007). In addition to the importance of iron in infant brain development, studies in South Africa have demonstrated that correction of iron deficiency can significantly improve maternal depression (Beard et al. 2005). Thus, iron uptake is important for growth and function of the brain, and it is

T. A. Rouault $(\bowtie) \cdot$ D.-L. Zhang $\cdot$ S. Y. Jeong

Molecular Medicine Program, National Institute of Child Health and Human Development,

National Institutes of Health, Bethesda, MD 20892, USA

e-mail: rouault@mail.nih.gov 
therefore important to understand how iron moves from the systemic circulation into the central nervous system.

Although the molecular details of brain iron uptake are incompletely characterized, many of the key proteins involved in uptake of dietary iron through the intestine have been characterized in the last decade. Identification of intestinal iron transporters represents an important advance, because once the biological problem of how to transport iron across a polarized epithelium has been solved, the same solution can be employed to resolve analogous challenges, including how to import iron into the central nervous system from the systemic circulation. More than $65 \%$ of iron is incorporated into heme in mammals, while numerous other proteins and enzymes also utilize iron as a cofactor, including tyrosine hydroxylase, which uses iron to convert tyrosine to dihydroxyphenylalanine in catechol synthesis. In addition, mitochondria require iron for function of numerous proteins, including aconitase, a key enzyme of the citric acid cycle, and respiratory chain complexes I-IV, which facilitate oxidative phosphorylation using several heme and iron-sulfur cluster cofactors (Rouault and Tong 2008). The central nervous system consumes about $20 \%$ of the total body energy (Drubach 1999) and is rich in iron; therefore, the mechanisms that govern brain iron uptake and homeostasis are of great interest.

The main transporters likely to be involved in brain iron uptake include proteins that are involved in transport of iron from the diet across the apical membrane of intestinal mucosal cells, as well as proteins that facilitate iron export from the basolateral membrane into the blood stream. A major protein involved in intestinal absorption of iron from the diet is a divalent metal transporter known as DMT1 (also known as Nramp2 and DCT1) (Mims and Prchal 2005), which imports iron into the intestinal epithelial cell (enterocyte), with the aid of a membrane-bound reductase that reduces ferric iron $(\mathrm{Fe} 3+)$ to the transportable ferrous form $(\mathrm{Fe} 2+)$ known as Dcytb (McKie 2008). It is not known how iron traverses the polarized enterocytes, but an iron exporter known as ferroportin (FPN) mediates iron export from the enterocytes into the circulation. FPN is the only mammalian iron exporter that has thus far been identified, and its substrate is ferrous (Fe2+) iron (Anderson and Vulpe 2009). In enterocytes, a membrane bound ferroxidase, hephaestin, oxidizes the iron transported by FPN, which enhances the rate of iron export and generates the ferric form of iron, which is sequentially accepted by transferrin (Tf) in the circulation (Wessling-Resnick 2006). Serum Tf is an abundant circulating protein that binds ferric iron and delivers iron to cells throughout the systemic circulation by binding to Tf receptors (TfRs) on cells in tissues such as liver and bone marrow. Upon binding, the Tf-TfR complex internalizes to a vesicle, iron is released from Tf, reduced to the transportable ferrous form $(\mathrm{Fe} 2+)$ by a vesicular reductase, Steap3 (Ohgami et al. 2006) and transported across the endosomal membrane into cytosol by DMT1 found in the vesicular membrane.

Intestinal iron uptake is highly regulated by a hormone known as hepcidin (Anderson et al. 2009), which is released mainly from the liver in response to iron overload or inflammation. Hepcidin can bind to FPN and induce its internalization and degradation, thereby blocking the iron uptake in response to systemic iron abundance. Intestinal iron uptake is also regulated by hypoxia inducible factor 2 (HIF2), a transcription factor known mainly for its ability to mediate the response of cells to hypoxia, through regulating the transcription of DMT1 and Dcytb, 
(Mastrogiannaki et al. 2009; Shah et al. 2009). Iron is stored intracellularly in the cytosolic protein ferritin, and major repositories of iron in the systemic circulation include the liver and the reticuloendothelial macrophages. All tissues in the body share a common system of iron delivery and homeostasis, based on intestinal iron uptake and circulating Tf, except for two regions that are separated from the systemic circulation by epithelial or endothelial barriers: the central nervous system, and testes (Rouault and Cooperman 2006).

The central nervous system (CNS) is separated from the systemic circulation by the blood-brain barrier, which is formed by endothelial cells of capillaries, and the blood-cerebrospinal fluid barrier, generated by epithelial cells found in the choroid plexus. Capillaries in the CNS are unusual in that they lack the fenestrations that characterize capillaries of the systemic circulation. Instead, tight junctions connect the endothelial cells in CNS capillaries, and the membrane composition of the portion of the cell that contacts blood differs from that of the basolateral surface of the cell, which is in contact with the brain interstitial fluid. Because there are no fenestrations, drugs and nutrients cannot pass between these cells, but rather must be transported across cells. Similarly, the choroid plexus consists of tuft-like capillaries that are ensheathed by an epithelium in which adjacent cells are joined by tight junctions that create the blood-cerebrospinal fluid (CSF) barrier. The choroid plexus protrudes into all four ventricles of the brain, and produces the CSF that fills the ventricles and bathes the interstitium of the central nervous system. Although the capillaries of the choroid plexus are fenestrated and allow substances to pass between cells, the choroid plexus epithelium presents a barrier through which nutrients and drugs must pass (reviewed in Rouault and Cooperman 2006).

The brain can be considered to consist of four major fluid compartments, and understanding the relationships among these compartments is important for comprehending the mechanisms involved in uptake of nutrients and drugs. One compartment consists of the intracellular fluid of the major cell types of the brain, including neurons, astrocytes, oligodendrocytes and microglia; the intracellular fluid of each cell can be reached only when nutrients or drugs cross the plasma membranes of each individual cell, either by passive diffusion or active uptake. The plasma membranes of these cells enable the cells to exclude many substances that are present in CSF, and to regulate their uptake of other nutrients and molecules as needed, usually because of the activity of dedicated transport molecules. The blood that flows through the brain represents a second compartment that is physically separated from the brain parenchyma by two cellular barriers: the blood brain barrier and the blood-cerebrospinal fluid (CSF) barrier. Cells of the choroid plexus elaborate $\mathrm{CSF}$, and substances that cross the choroidal epithelium or blood brain barrier enter into the ventricles or brain interstitial fluid (Brown et al. 2004). Although the CSF and interstitial fluid have been characterized as a third and fourth compartment (Zheng et al. 2003), there is no diffusional barrier that separates brain interstitial fluid from ventricular CSF, and once a substance has entered the interstitial fluid or ventricular fluid, it may freely diffuse to the membranes of all cells within the brain interstitium. There is a net flow of CSF from its point of synthesis in the choroid plexus, which extends like a net throughout all four ventricles and closely apposes important brain regions such as the hippocampus, to several locations where CSF is 
thought to drain into the venous circulation (Koh et al. 2007), including the arachnoid villi, and also the cribriform plate (Johansson et al. 2008).

The choroid plexus has not previously been considered to be very important in maintenance of brain iron homeostasis, in part because the blood brain barrier has been calculated to provide much more surface area than the choroid plexus (Moos et al. 2007). However, the potential role of the choroid plexus may be greatly underestimated for several reasons. Although the choroid plexus apical surface area was previously estimated to be less than half that of the blood brain barrier (Keep and Jones 1990), more recent studies suggest that the total surface area provided by microvilli on both the apical and basolateral membranes of the choroid plexus cells is ten-fold greater than was previously estimated (Speake et al. 2004). Moreover, the blood flow rate to the capillaries of the choroid plexus is about five-fold higher than to other regions of the brain (Maktabi et al. 1990), and the combination of high blood supply and high surface area increases the possibility that the choroid plexus plays an important role in uptake of nutrients such as iron. In uptake studies that involve brain dissection, the delicate net-like choroid plexus structures are frequently torn or lost, which may contribute to under-appreciation of the broad physical contact between the choroid plexus and ventricular system. In contrast, live imaging studies demonstrate that the choroid plexus structures spread throughout the ventricles, pervading into the far-reaches of the ventricular system (Naeini et al. 2009).

Iron uptake into the brain is high in infants when the brain is growing and developing, but measurable iron uptake is lower in adult, and it was previously believed that most iron uptake needed for brain development occurred passively in infants because the blood brain barrier and blood CSF barrier were not yet fully formed (Berg et al. 2001). However, numerous studies indicate that the blood and CSF barriers function very early in development (Johansson et al. 2008), and it is not likely that all of the iron the brain will need during growth and development is taken up before barriers begin to function. In addition, studies over the last ten years have revealed that TfRs are present on the luminal membranes of capillary endothelial cells, and that iron from systemic Tf can cross endothelial cells and enter the interstitium, even though $\mathrm{Tf}$ itself does not cross from the systemic circulation into the interstitial fluid (Morgan and Moos 2002; Moos et al. 2006). These studies indicate that iron can enter into the brain interstitium in adult animals. Consistent with these observations, other proteins identified as important in iron transport in the duodenum, including the iron exporter, FPN (Wu et al. 2004) and the ferroxidases, hephaestin and ceruloplasmin, have been identified in brain vasculature, although not all of the proteins involved in transport, such as DMT1, have been identified in capillaries (Moos et al. 2007). Detection of some iron transport proteins led to focus on the blood brain barrier as a major point through which iron passes into the CNS, particularly because the surface area of the blood brain barrier is extensive (Moos 2002; Moos et al. 2007).

However, it is difficult to directly measure brain iron uptake and ascertain the relative importance of brain capillaries compared to the choroid plexus, because there are no real-time imaging techniques that permit non-invasive overall visualization of iron uptake. Most studies have been performed in animal models using radioactively labeled iron isotopes, and iron distribution in the CNS has been 
visualized by autoradiograms performed at specific times after injection of radiolabeled iron in sacrificed animals. In contradistinction to iron, uptake of manganese, a divalent metal, can be visualized directly in magnetic resonance imaging because of its particular magnetic properties. After a bolus injection of manganese in the systemic circulation, studies indicate that early manganese uptake can be visualized in the choroid plexus within five minutes after injection (Aoki et al. 2004). Within ten minutes, manganese had diffused into ventricular fluid, and within several hours, manganese was visible within the peri-ventricular interstitial spaces. After 24 hours, uptake in neurons and glia was visualized, and the pattern of distribution remained unchanged for several weeks, after which time manganese slowly exited the CNS, possibly through either the arachnoid villi or the cribriform plate, another mode of egress that has been described recently (Johansson et al. 2008).

Since manganese shares some chemical features with iron and may be transported by some of the same basic transporters, its route of uptake into the CNS may also be used by iron. To avoid the problems associated with radiotracer studies, which involve timed sacrifice of animals, and to avoid bias based on expectations, we decided to evaluate the expression levels of known iron transporters in the CNS using the technique of in situ hybridization, which identifies sites where genes are highly expressed by quantitatively labeling messenger RNAs that encode proteins of interest. Over the last several years, the Allen Brain atlas, an online resource, has systematically compiled the results of in situ hybridizations for all known mouse genes, and determined their sites of expression. The results of these experiments are unbiased, as they are part of an encyclopedic compendium of results (Lein et al. 2007). Thus, elucidation of where brain iron transporters are expressed revealed by in situ hybridization results has the potential to reorient expectations about the major sites of iron transport.

We analyzed expression patterns of iron transporters that were identified originally in the duodenal mucosa, including the apical transporter DMT1, the ferric reductase, Dcytb, the basolateral exporter ferroportin, and the membrane bound ferroxidase hephaestin. In addition, a special form of the normally soluble ferroxidase, ceruloplasmin, is found in the brain; it differs from ceruloplasmin in the systemic circulation because it contains a glycolipid modification at the C-terminus known as a glycophosphatidylinositol (GPI) anchor, which anchors the ceruloplasmin to membranes of astrocytes and possibly other cell types within the CNS (Patel et al. 2000). Although immunohistochemistry has been most commonly used to ascertain where specific proteins are expressed in the central nervous system, in situ hybridization is a complementary technique that also addresses the question of where genes are highly expressed.

Iron that enters the CNS should be delivered to barrier cells by the circulating iron-bearing Tf. Under normal physiological conditions there is almost no free iron in circulation. Upon binding the TfR, the Tf-TfR complex usually internalizes to endosome, where iron is released from $\mathrm{Tf}$, reduced from the ferric $(\mathrm{Fe} 3+)$ to the ferrous $(\mathrm{Fe} 2+$ ) form, exported to cytosol by DMT1, and then exported out of the cell by FPN, with the aid of ferroxidases such as ceruloplasmin and hephaestin. Upon reaching the interstitial fluid of the brain, the iron can be bound by $\mathrm{Tf}$ that was synthesized by oligodendrocytes and released directly into the interstitial fluid. A Tfiron complex can then form and circulate throughout the brain interstitium, supplying iron to cells within the CNS (reviewed in Zecca et al. 2004). 


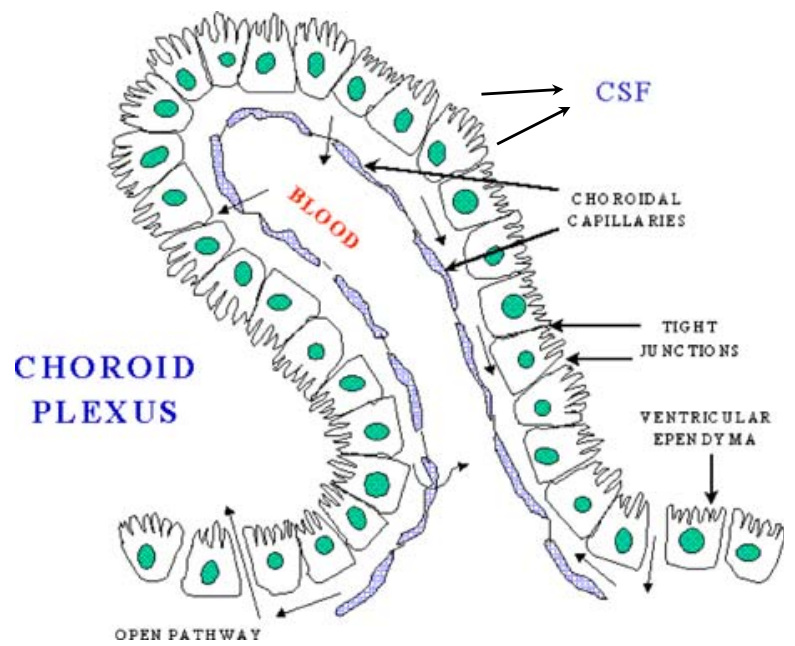

Fig. 1 A schematic of the choroid plexus illustrates that the choroidal capillaries are separated from the CSF by a layer of choroidal epithelial cells. If the choroid plexus acted as a site of brain iron uptake, Tf released by the blood would bind to TfR on the membrane nearest to the capillary, and iron would cross to the membrane that faces the CSF, and be exported by FPN, aided by a ferroxidase such as ceruloplasmin or hephaestin. In this potential scheme, DMT1 would be needed to release iron from endosomes after internalization of the Tf-TfR complex, and an endosomal reductase would also be needed. This figure was reproduced from http://www.daviddarling.info/encyclopedia/copyright.html with permission from David Darling

A schematic figure of the choroid plexus depicted in Fig. 1 shows the relationship of the fenestrated vasculature to the epithelial layer of the choroid plexus and to CSF. As discussed in the legend, iron that enters the CNS would be expected to be delivered to TfRs on barrier cells by circulating Tf, which carries ferric iron. TfR1 was well represented in choroid plexus on in situ analysis, but its expression was

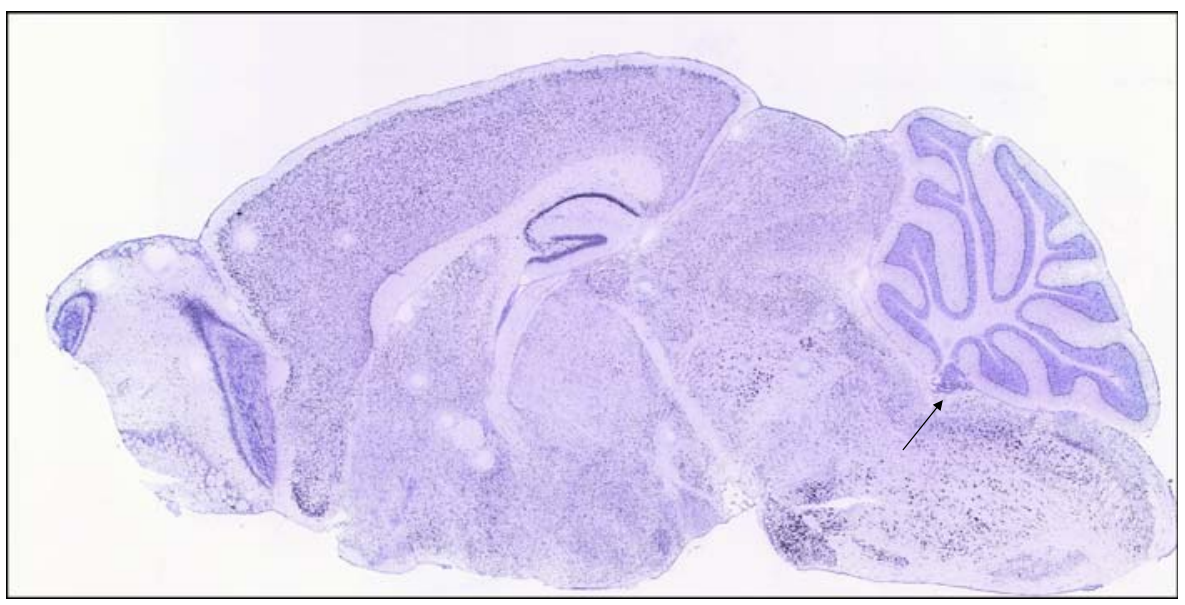

Fig. 2 The transferrin receptor is expressed throughout the central nervous system, including in the choroid plexus, as judged by staining in a sagittal section of mouse brain from the Allen Mouse Brain atlas (arrow) 


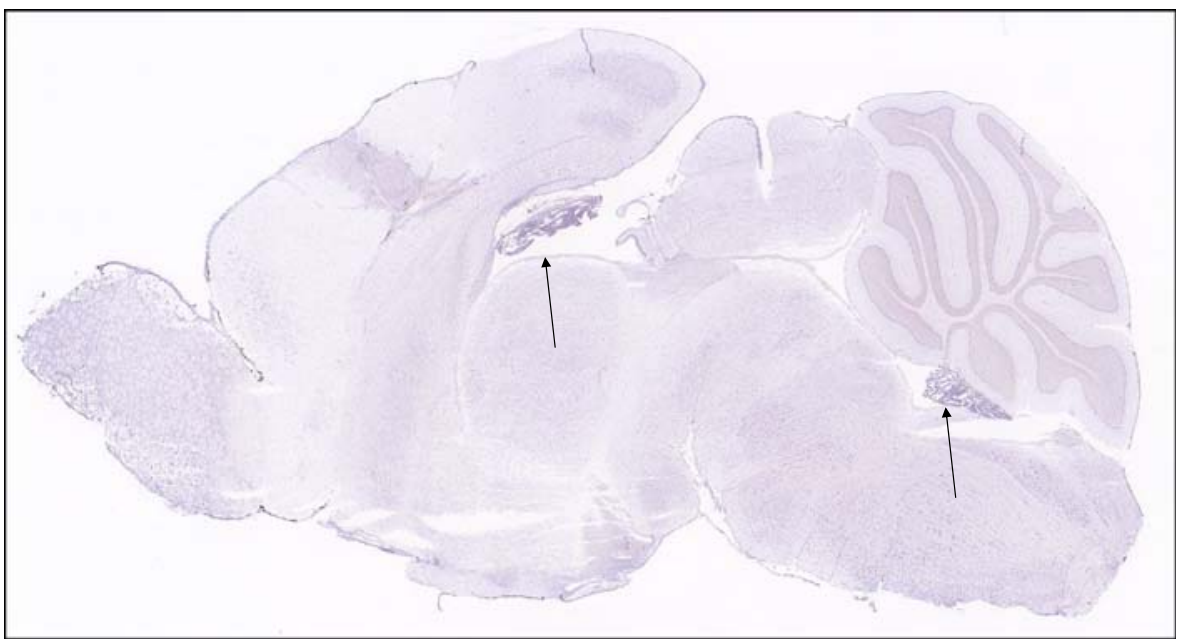

Fig. 3 Expression of the divalent metal transporter, DMT1, is notably high in the choroid plexus, as indicated by arrows to choroid plexus formations

also high in many other regions of the brain (Fig. 2). Strikingly, expression of DMT1, FPN, ceruloplasmin (Cp), and hephaestin were higher in the choroid plexus on in situ hybridization than virtually all other brain regions (Figs. 3, 4, 5, 6 and 7). Notably, these transport proteins were not detected in capillaries, even though expression of the known capillary protein, Epas1, also known as Hif2 alpha, can be readily detected in the Allen Brain atlas (data not shown). Also, the expression of the ferric reductase, Dcytb, was substantial in choroid plexus (Fig. 4), and that result was further supported by immunohistochemical detection of the protein (Fig. 10).

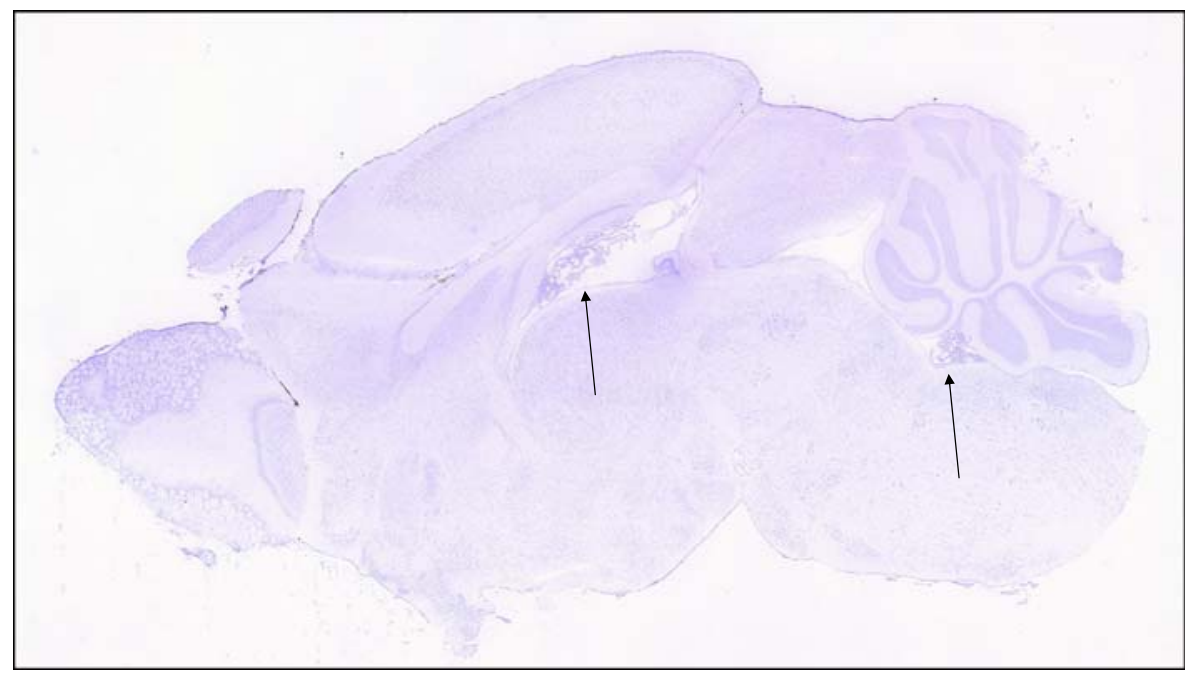

Fig. 4 Expression of the reductase, Dcytb, is detectable in many regions of the brain, including in the choroid plexus (arrows) 


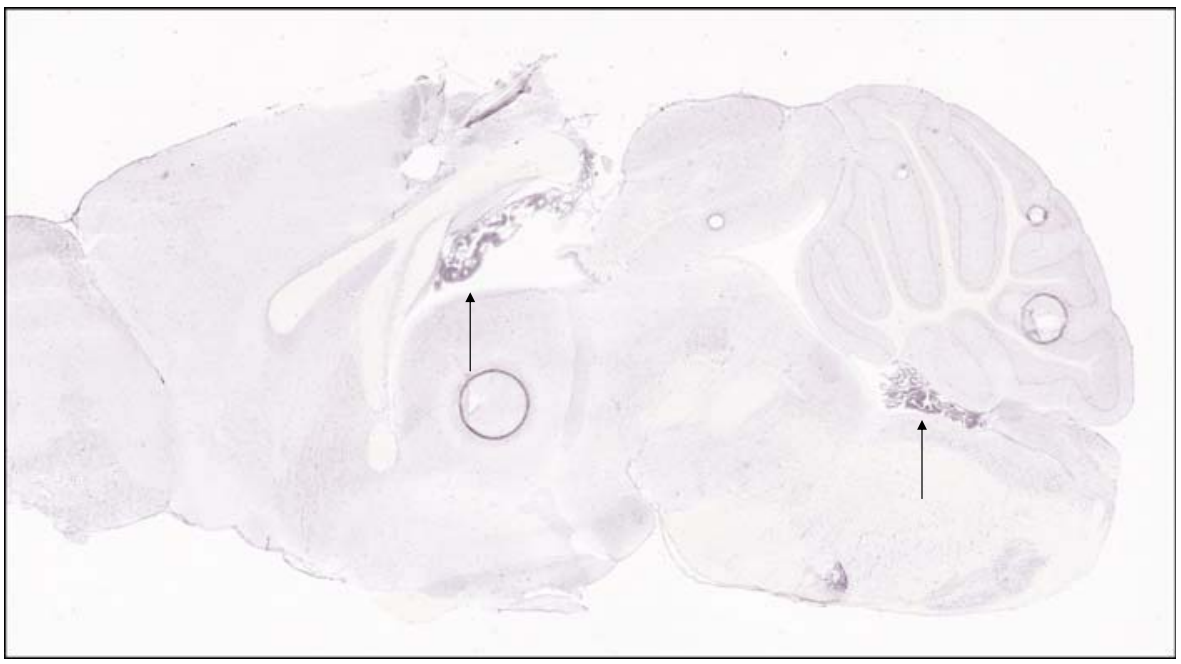

Fig. 5 Ferroportin expression is very high in choroid plexus compared to other regions of the CNS (arrows)

Iron strorage protein ferritin can be used as a surrogate iron marker since its expression is regulated by intracellular iron level. Messenger RNA expression of ferritin $\mathrm{H}$ chain was also high in choroid plexus compared to other areas (Fig. 8) and this was confirmed by immunofluorescent assay (Fig. 9a). Interestingly, there was massive increase of

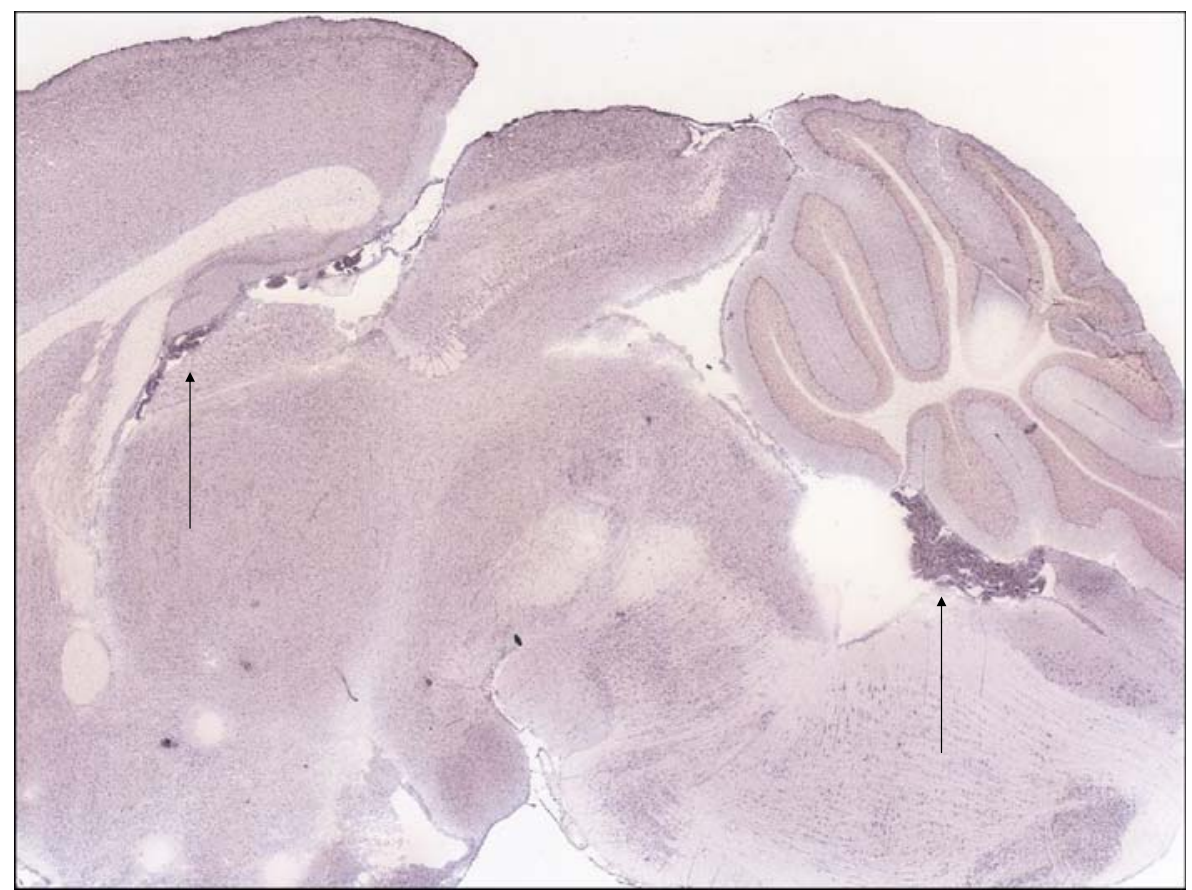

Fig. 6 Expression of the membrane-bound ferroxidase, hephaestin, is detectable throughout the brain, but is very high in the choroid plexus (arrows) 


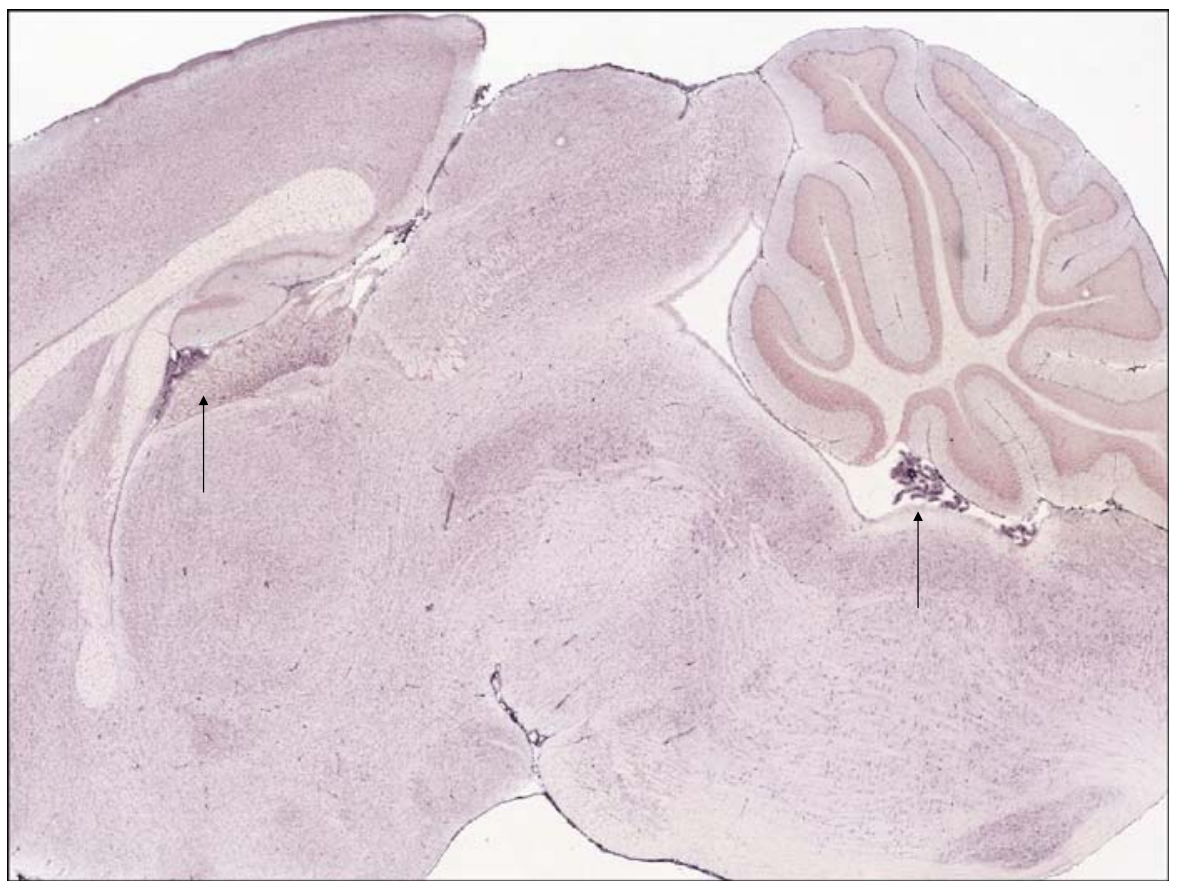

Fig. 7 Expression of ceruloplasmin is also very high in the choroid plexus (arrows)

ferritin expression in the 24 months old Cp null mice (Fig. 9c), which show abnormal iron accumulation as they age (Jeong and David 2006) (Fig. 10).

Taken together, these results from in situ hybridization point to the possibility that the choroid plexus may mediate significant iron transport in the mammalian central

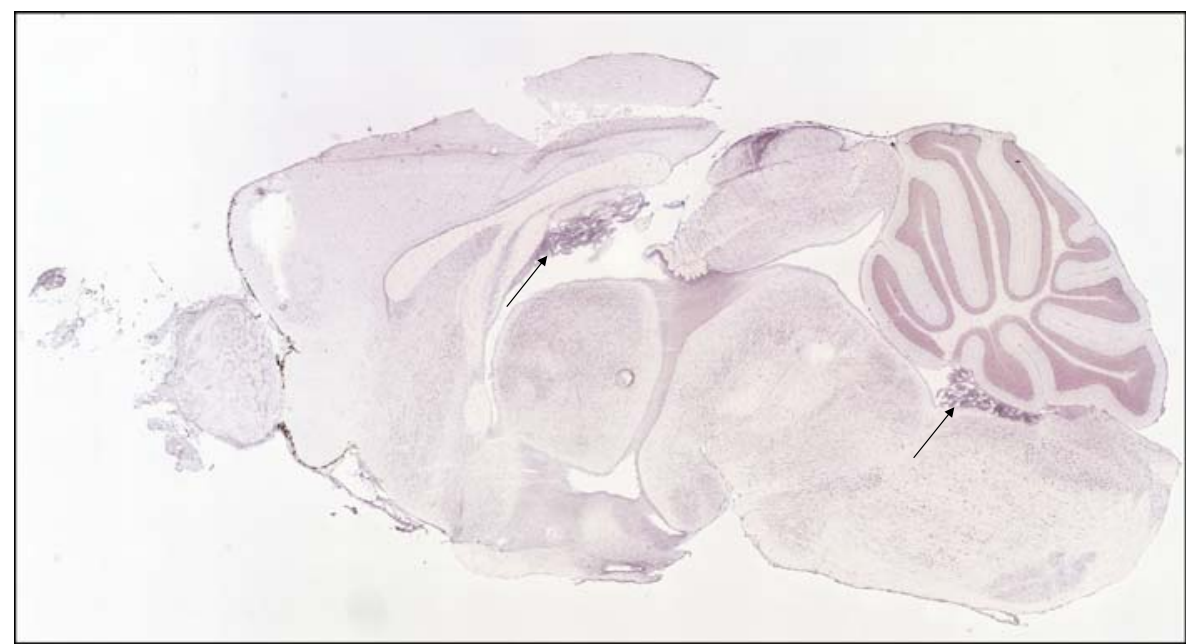

Fig. 8 Expression of iron storage protein, ferritin $\mathrm{H}$ chain is highly expressed in the choroids plexus (arrows) 


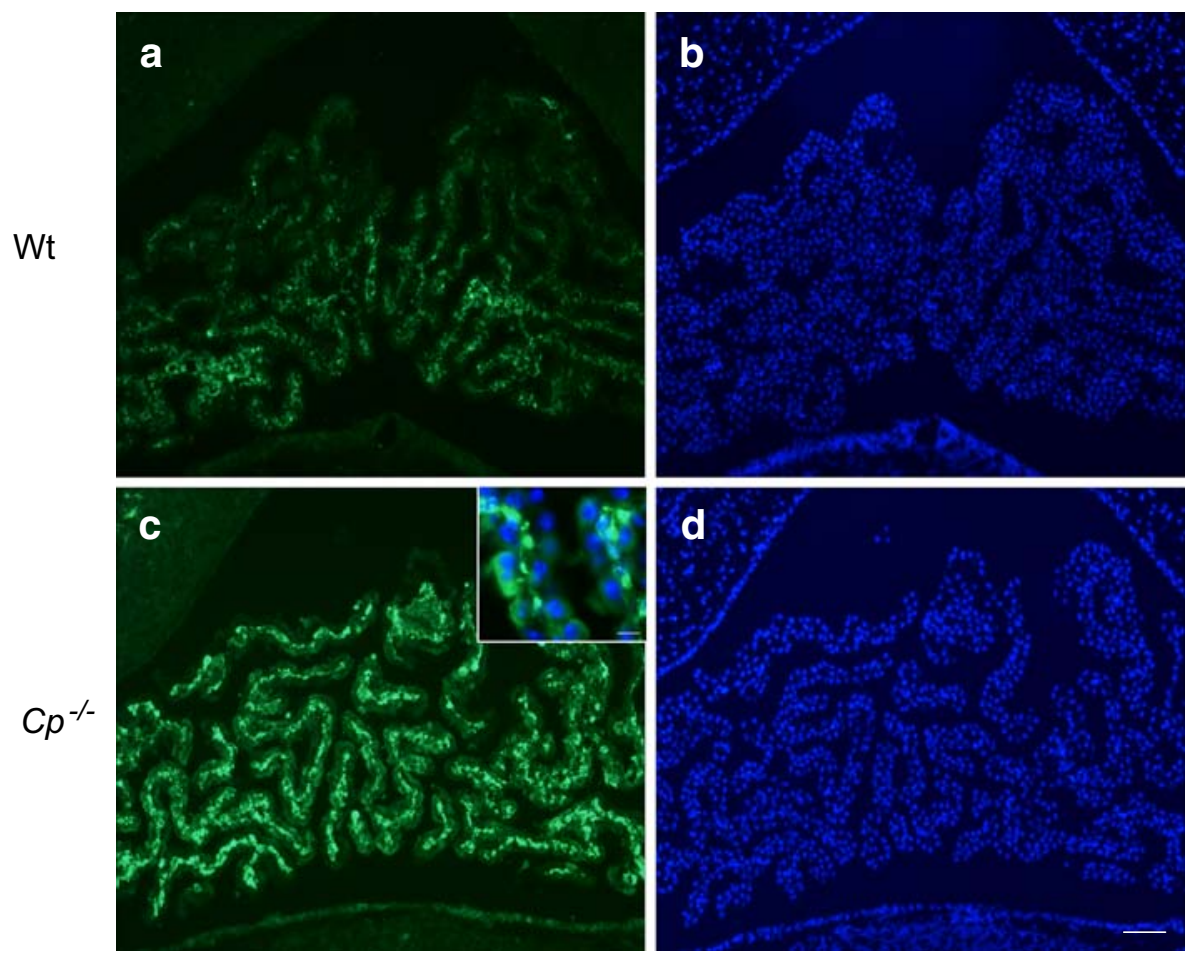

Fig. 9 Immunofluorescent staining using anti-ferritin antibody (Dako) shows expression of ferritin in 24months old mice choroid plexus (a). This immunoreactivity is highly increased in ceruloplasmin null mice (c) indicating increased intracellular iron in this area. DAPI staining $\mathbf{b}$, $\mathbf{d}$ shows choroid plexus structure near fourth ventricle. Scale bars; $100 \mu \mathrm{m}, 20 \mu \mathrm{m}$ (inset)

Fig. 10 Immunohistochemistry reveals high expression of Dcytb in the choroid plexus epithelial cells, indicated by the green staining. Nuclei of epithelial, capillary and other cells are detected by staining with DAPI blue. Immunohistochemistry was performed on the frozen section of $10 \mu \mathrm{m}$ thickness with a rabbit anti-mouse Dcytb polyclonal antibody as described previously (Su et al. 2006; Zhang et al. 2006)

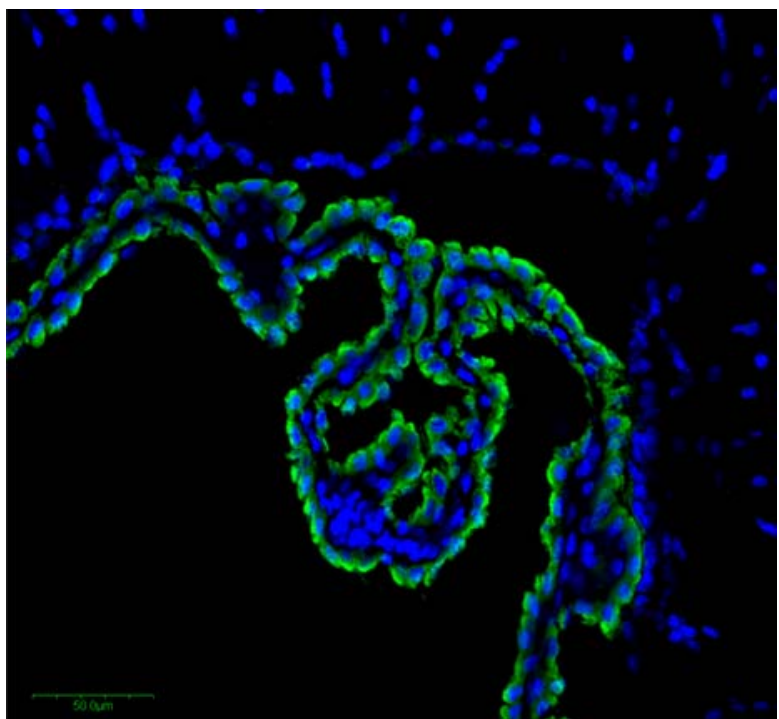


nervous system. The proposed pathway of uptake through the choroid plexus has already been demonstrated to function in manganese uptake (Aoki et al. 2004), but imaging techniques that allow real-time visualization of iron uptake have not been developed. Brain iron uptake is a critical process that must be highly regulated. If the choroid plexus is a major site of iron uptake, then diseases that cause choroid plexus inflammation could have adverse effects on brain iron homeostasis. Recent studies support an important role for the choroid plexus in iron homeostasis and in response to systemic inflammation (Marques et al. 2009). We suggest that analysis of in situ hybridization results that are available from the Allen Brain Institute may facilitate discovery of previously unappreciated uptake pathways for a variety of brain nutrients and drugs.

Acknowledgments This work was supported by the intramural program of the National Institute of Child Health and Human Development, Bethesda, MD. We thank David Darling for permission to use the choroid plexus schematic in Fig. 1. We are grateful to Dr. Samuel David for the permission to use ceruloplasmin null mice data. We also thank the Allan Brain Institute for use of their online materials. In situ hybridization figures were obtained from the Allen Mouse Brain Atlas [Internet]. Seattle (WA): Allen Institute for Brain Science. C2009. Available from: http://mouse.brain-map.org.

Open Access This article is distributed under the terms of the Creative Commons Attribution Noncommercial License which permits any noncommercial use, distribution, and reproduction in any medium, provided the original author(s) and source are credited.

\section{References}

Anderson GJ, Vulpe CD (2009) Mammalian iron transport. Cell Mol Life Sci 66:3241-3261

Anderson GJ, Frazer DM, McLaren GD (2009) Iron absorption and metabolism. Curr Opin Gastroenterol 25:129-135

Aoki I, Wu YJ, Silva AC, Lynch RM, Koretsky AP (2004) In vivo detection of neuroarchitecture in the rodent brain using manganese-enhanced MRI. Neuroimage 22:1046-1059

Beard JL, Hendricks MK, Perez EM, Murray-Kolb LE, Berg A, Vernon-Feagans L, Irlam J, Isaacs W, Sive A, Tomlinson M (2005) Maternal iron deficiency anemia affects postpartum emotions and cognition. J Nutr 135:267-272

Berg D, Gerlach M, Youdim MB, Double KL, Zecca L, Riederer P, Becker G (2001) Brain iron pathways and their relevance to Parkinson's disease. J Neurochem 79:225-236

Brown PD, Davies SL, Speake T, Millar ID (2004) Molecular mechanisms of cerebrospinal fluid production. Neuroscience 129:957-970

Drubach D (1999) The brain explained. Prentice Hall, Upper Saddle River, New Jersey

Jeong SY, David S (2006) Age-related changes in iron homeostasis and cell death in the cerebellum of ceruloplasmin-deficient mice. J Neurosci 26:9810-9819

Johansson PA, Dziegielewska KM, Liddelow SA, Saunders NR (2008) The blood-CSF barrier explained: when development is not immaturity. Bioessays 30:237-248

Keep RF, Jones HC (1990) A morphometric study on the development of the lateral ventricle choroid plexus, choroid plexus capillaries and ventricular ependyma in the rat. Brain Res Dev Brain Res 56:47-53

Koh L, Nagra G, Johnston M (2007) Properties of the lymphatic cerebrospinal fluid transport system in the rat: impact of elevated intracranial pressure. J Vasc Res 44:423-432

Lein ES et al (2007) Genome-wide atlas of gene expression in the adult mouse brain. Nature 445:168-176

Maktabi MA, Heistad DD, Faraci FM (1990) Effects of angiotensin II on blood flow to choroid plexus. Am J Physiol 258:H414-H418

Marques F, Falcao AM, Sousa JC, Coppola G, Geschwind D, Sousa N, Correia-Neves M, Palha JA (2009) Altered iron metabolism is part of the choroid plexus response to peripheral inflammation. Endocrinology 150:2822-2828 
Mastrogiannaki M, Matak P, Keith B, Simon MC, Vaulont S, Peyssonnaux C (2009) HIF-2alpha, but not HIF-1alpha, promotes iron absorption in mice. J Clin Invest 119:1159-1166

McKie AT (2008) The role of Dcytb in iron metabolism: an update. Biochem Soc Trans 36:1239-1241

Mims MP, Prchal JT (2005) Divalent metal transporter 1. Hematology 10:339-345

Moos T (2002) Brain iron homeostasis. Dan Med Bull 49:279-301

Moos T, Skjoerringe T, Gosk S, Morgan EH (2006) Brain capillary endothelial cells mediate iron transport into the brain by segregating iron from transferrin without the involvement of divalent metal transporter 1. J Neurochem 98:1946-1958

Moos T, Rosengren Nielsen T, Skjorringe T, Morgan EH (2007) Iron trafficking inside the brain. J Neurochem 103:1730-1740

Morgan EH, Moos T (2002) Mechanism and developmental changes in iron transport across the bloodbrain barrier. Dev Neurosci 24:106-113

Naeini RM, Yoo JH, Hunter JV (2009) Spectrum of choroid plexus lesions in children. AJR Am J Roentgenol 192:32-40

Ohgami RS, Campagna DR, McDonald A, Fleming MD (2006) The Steap proteins are metalloreductases. Blood 108:1388-1394

Patel BN, Dunn RJ, David S (2000) Alternative RNA splicing generates a glycosylphosphatidylinositolanchored form of ceruloplasmin in mammalian brain. JBC 275:4305-4310

Rouault TA, Cooperman S (2006) Brain iron metabolism. Semin Pediatr Neurol 13:142-148

Rouault TA, Tong WH (2008) Iron-sulfur cluster biogenesis and human disease. Trends Genet 24:398-407

Shah YM, Matsubara T, Ito S, Yim SH, Gonzalez FJ (2009) Intestinal hypoxia-inducible transcription factors are essential for iron absorption following iron deficiency. Cell Metab 9:152-164

Speake T, Kibble JD, Brown PD (2004) Kv1.1 and Kv1.3 channels contribute to the delayed-rectifying K+ conductance in rat choroid plexus epithelial cells. Am J Physiol Cell Physiol 286:C611-C620

Su D, May JM, Koury MJ, Asard H (2006) Human erythrocyte membranes contain a cytochrome b561 that may be involved in extracellular ascorbate recycling. J Biol Chem 281:39852-39859

Walker SP et al (2007) Child development: risk factors for adverse outcomes in developing countries. Lancet 369:145-157

Wessling-Resnick M (2006) Iron imports. III. Transfer of iron from the mucosa into circulation. Am J Physiol Gastrointest Liver Physiol 290:G1-G6

Wu LJ et al (2004) Expression of the iron transporter ferroportin in synaptic vesicles and the blood-brain barrier. Brain Res 1001:108-117

Youdim MB (2008) Brain iron deficiency and excess; cognitive impairment and neurodegeneration with involvement of striatum and hippocampus. Neurotox Res 14:45-56

Zecca L, Youdim MB, Riederer P, Connor JR, Crichton RR (2004) Iron, brain ageing and neurodegenerative disorders. Nat Rev Neurosci 5:863-873

Zhang DL, Su D, Berczi A, Vargas A, Asard H (2006) An ascorbate-reducible cytochrome b561 is localized in macrophage lysosomes. Biochim Biophys Acta 1760:1903-1913

Zheng W, Aschner M, Ghersi-Egea JF (2003) Brain barrier systems: a new frontier in metal neurotoxicological research. Toxicol Appl Pharmacol 192:1-11 